\title{
INSTRUMENTOS DE AVALIAÇÃO E PARÂMETROS DE QUALIDADE PARA A EDUCAÇÃO INFANTIL
}

\author{
Dirce Guerres-ZuccoI \\ Andreia Zanella II \\ (D) Angela Scalabrin Coutinho III \\ I Prefeitura Municipal de Florianópolis, Florianópolis (SC), Brasil; dirceguerreszucco@gmail.com \\ II Universidade Federal de Santa Catarina (UFSC), Florianópolis (SC), Brasil; andreia.zanella@ufsc.br \\ III Universidade Federal do Paraná (UFPR), Curitiba (PR), Brasil; angelamscoutinho@gmail.com
}

\section{Resumo}

O artigo, de abordagem qualitativa, trata de instrumentos de avaliação e parâmetros de qualidade para a educação infantil. Propõe-se a identificar, descrever e comparar diferentes modelos de avaliação da qualidade da educação infantil, analisando sua adequação para aplicação na rede municipal de ensino de Florianópolis, Santa Catarina, Brasil. Dentre os modelos analisados, dois mostraram maior coerência teórico-metodológica ao contexto, por se basearem no acesso, insumos e processos e preverem a participação da comunidade educativa. A implementação e gestão de um sistema de avaliação requer indissociabilidade entre política de avaliação e instrumentos de avaliação por meio da intersetorialidade, fomentados por políticas públicas para infância a fim de atender aos parâmetros de qualidade da educação infantil.

AVALIAÇÃO DA EDUCAÇÃO • QUALIDADE DA EDUCAÇÃO • EDUCAÇÃO INFANTIL

\section{EVALUATION INSTRUMENTS AND QUALITY PARAMETERS FOR EARLY CHILDHOOD EDUCATION}

\begin{abstract}
The article, with a qualitative approach, deals with evaluation instruments and quality parameters for early childhood education. It is proposed to identify, describe and compare different models for assessing the quality of early childhood education, analyzing their suitability for application in the public education system of Florianópolis, Santa Catarina, Brazil. Among the models analyzed, two showed greater theoretical and methodological coherence to the context, because they are based on access, inputs and processes and provide for the participation of the educational community. The implementation and management of an evaluation system requires an inseparability between evaluation policy and evaluation instruments through intersectionality, fostered by public policies for children to meet the quality parameters of early childhood education.
\end{abstract}




\section{INSTRUMENTOS DE EVALUACIÓN Y PARÁMETROS DE CALIDAD PARA LA EDUCACIÓN INFANTIL}

\section{Resumen}

El artículo, de enfoque cualitativo, trata sobre los instrumentos de evaluación y los parámetros de calidad de la educación infantil. Se propone identificar, describir y comparar diferentes modelos para evaluar la calidad de la educación infantil, analizando su adecuación para su aplicación en el sistema municipal de educación de Florianópolis, Santa Catarina, Brasil. Entre los modelos analizados, dos mostraron mayor coherencia teórico-metodológica al contexto, pues se basan en accesos, insumos y procesos y predicen la participación de la comunidad educativa. La implementación y gestión de un sistema de evaluación requiere la inseparabilidad entre la política de evaluación y los instrumentos de evaluación a través de la intersectorialidad, fomentada por políticas públicas para la niñez a fin de cumplir con los parámetros de calidad de la educación infantil.

\section{EVALUACIÓN DE LA EDUCACIÓN • CALIDAD DE LA EDUCACIÓN • EDUCACIÓN DE LA PRIMERA INFANCIA}

\section{OUTILS D'ÉVALUATION ET PARAMÈTRES DE QUALITÉ POUR L'ÉDUCATION DE LA PETITE ENFANCE}

\section{Résumé}

À travers une approche qualitative, cet article aborde des instruments d'évaluation et des paramètres de qualité pour l'éducation de la petite enfance. Il vise à identifier, décrire et comparer différents modèles d'évaluation concernant la qualité de l'éducation de la petite enfance, en analysant leur potentiel d'applicabilité dans le réseau municipal d'éducation de Florianópolis, Santa Catarina, Brasil. Parmi tous les modèles analysés, deux ont présenté une plus grande cohérence théorique et méthodologique avec le contexte, non seulement du point de vue de l'accès, des intrants et des processus, mais aussi parce qu'ils prévoient la participation de la communauté éducative. La mise en œuvre et la gestion d'un système d'évaluation reposent sur une indissociabilité entre politique et instruments d'évaluation par le biais de l'intersectorialité et doivent être stimulés par les politiques publiques pour l'enfance afin de se conformer aux paramètres de qualité de l'éducation de la petite enfance.

ÉVALUATION DE L'ÉDUCATION • QUALITÉ DE L'ÉDUCATION • ÉDUCATION DE LA PETITE ENFANCE 


\section{A ÚLTIMA DÉCADA, A AVALIAÇÃO DA QUALIDADE DA EDUCAÇÃO INFANTIL (EI) TEM SIDO TEMA}

central da política educacional, apresentando-se em diversos contextos como estratégia para o levantamento de dados sobre a qualidade dos serviços. Precedido pelos Parâmetros nacionais de qualidade para a educação infantil (Ministério da Educação [MEC], 2006), foi publicado, também pelo MEC (2009), o documento Indicadores da qualidade na educação infantil. Segundo o monitoramento realizado pelo MEC (2011), verificou-se que a maioria dos municípios que utilizavam o instrumento era de pequeno porte, com finalidades variadas, entre estas, o diagnóstico das redes. Na rede municipal de ensino (RME) de Florianópolis, os Indicadores da qualidade na educação infantil (MEC, 2009) tiveram uma adesão distinta nas unidades educativas, variando conforme o incentivo e acompanhamento por parte da Diretoria de Educação Infantil (DEI) e o fomento da equipe diretiva.

No bojo das ações, em âmbito federal, em torno da avaliação da qualidade da EI, destaca-se ainda a publicação da Portaria n. 1.147 (2011), que propôs a formação de um Grupo de Trabalho (GT), coordenado pela Secretaria de Educação Básica (SEB) e constituído por especialistas representantes do MEC, das universidades, de movimentos sindicais, sociais, entre outros. O trabalho realizado pelo GT resultou na publicação do documento Educação infantil: Subsídios para a construção de uma sistemática de avaliação (MEC, 2012), evidenciando diretrizes e subsídios sem padronizar ou indicar um instrumento de avaliação específico.

Diante da eminente demanda para o levantamento de dados sobre a qualidade da EI, a segunda edição do Plano Nacional de Educação (PNE) estabelece metas e estratégias, dentre as quais a realização periódica da avaliação a cada dois anos, a fim de aferir a qualidade da EI, conforme os parâmetros nacionais de qualidade, além de direcionar as políticas públicas para a etapa (Lei n. 13.005, 2014, p. 2).

Em 2019, e não em 2016 como previa o PNE, o Instituto Nacional de Estudos e Pesquisas Educacionais Anísio Teixeira (Inep) realizou a avaliação nacional da EI como um estudo piloto em cumprimento à Portaria n. 271 (2019). Essa avaliação se constituiu de dados levantados no Censo Escolar, o que já ocorria, e em questionários respondidos por gestores das unidades educacionais e das redes de ensino e por, pelo menos, um docente de cada unidade de EI dos municípios participantes.

Quanto à avaliação da EI na perspectiva do monitoramento, em 2009, Florianópolis e outras cinco capitais brasileiras (Belém, Campo Grande, Fortaleza, Rio de Janeiro e Teresina) foram selecionadas para mapear a qualidade do atendimento, por meio do modelo norte-americano composto pelas escalas Infant Toddler Environment Rating Scale (Iters-R) - Escala de Avaliação do Ambiente Infantil - e Early Childhood Environment Rating Scale (Ecers-R) Escala de Avaliação do Ambiente na Primeira Infância -, ambas na versão revisada. Na ocasião participaram da avaliação 147 instituições de educação infantil, das quais 30 faziam parte da RME de Florianópolis. A avaliação foi desenvolvida em parceria com a Fundação Carlos Chagas, o Ministério da Educação e o Banco Interamericano de Desenvolvimento (Campos et al., 2011, p. 20). Em 2015, a capital catarinense aplicou novamente a avaliação utilizando o modelo norte-americano, mas desta vez com abrangência censitária, com a participação das 87 unidades educativas da RME.

Outra experiência avaliativa realizada no município, entre 2013 e 2015, foi baseada nas escalas italianas Indicatori e Scala della Qualità Educativa del Nido (Isquen) - Indicadores e Escala da Qualidade Educativa da Creche - e Autovalutazione della Scuola dell'Infanzia (AVSI) - Autoavaliação da Pré-escola. Essa avaliação fundamentou-se em uma perspectiva de pesquisa 
qualitativa, com o objetivo de discutir a potencialidade dos instrumentos italianos e os impactos teórico-metodológicos de uma avaliação de natureza reflexiva e participante (Souza et al., 2017, p. 23), atrelada ao projeto Formação em rede na educação infantil: Avaliação de contexto (Souza et al., 2015). Tal estudo foi desenvolvido no setor de Educação da Universidade Federal do Paraná (UFPR), representado pelas pesquisadoras Angela Coutinho, Gisele de Souza e Catarina Moro, com a consultoria das pesquisadoras italianas Anna Bondioli e Donatella Savio, da Università degli Studi di Pavia na Itália. O grupo coordenou a avaliação com o apoio de pesquisadores universitários de quatro capitais brasileiras - Curitiba, Belo Horizonte, Florianópolis e Rio de Janeiro -, contemplando a avaliação de uma instituição pública municipal.

Para o cumprimento da Política Nacional de Avaliação da Educação Básica, a Portaria n. 458 (2020) alterou o Sistema de Avaliação da Educação Básica (Saeb), ao deliberar que a avaliação da educação infantil seja de caráter censitário, com periodicidade anual, "tendo como objetivo aferir o domínio das competências e das habilidades esperadas ao longo da educação básica, de acordo com a Base Nacional Comum Curricular (BNCC) e as correspondentes diretrizes curriculares nacionais" (Portaria n. 458, 2020). No entanto, mais tarde, a Portaria n. 10 , de 8 de janeiro de 2021 , art. $5^{\circ}$, inciso $2^{\circ}$, altera a Portaria anterior e institui que "a EI será avaliada a cada dois anos exclusivamente pela aplicação de questionários eletrônicos de natureza não cognitiva" (Portaria n. 10, 2021). Quanto à abrangência, a Portaria n. 250, de 5 de julho de 2021, estabelece as diretrizes de realização do Saeb em 2021 e define que a avaliação da EI será de caráter amostral.

Diante de um cenário de incertezas, no que diz respeito à metodologia da avaliação, a preocupação paira em torno da especificidade da faixa etária de 0 a 5 anos - que apresenta princípios e diretrizes curriculares pautados nas brincadeiras, interações e linguagens - agregada à diversidade das instituições brasileiras. Ao considerar esses elementos para a construção, ou mesmo adaptação, de um instrumento de avaliação, esta pesquisa, de natureza qualitativa e aplicada, apresenta alguns resultados, a partir de seu objetivo geral, ao analisar e discutir a adequação dos modelos de avaliação da qualidade da educação infantil para a RME de Florianópolis, com a possibilidade de estender-se ao contexto da EI brasileira.

Quanto aos objetivos específicos, o trabalho se propôs a: identificar e categorizar os principais modelos de avaliação da qualidade da EI, tanto nacionais quanto estrangeiros; definir os parâmetros de qualidade que devem fazer parte de um instrumento de avaliação; comparar os instrumentos de avaliação, visando a identificar o alinhamento desses aos parâmetros de qualidade da EI; e discutir a adequação dos instrumentos já existentes para a avaliação da RME de Florianópolis, com a possibilidade de se estender ao contexto da EI brasileira.

Com base na literatura e na legislação, o artigo apresenta alguns parâmetros e concepções de avaliação da qualidade da EI. Para esse fim são elencados e categorizados instrumentos de avaliação, tanto nacionais quanto estrangeiros, descrevendo-os para posterior análise comparada. Por último, são mostrados os modelos que mais se aproximaram da realidade florianopolitana, considerando sua coerência metodológica, os princípios da educação infantil, a adaptação ao contexto, bem como a participação da comunidade educativa.

\section{Parâmetros da avaliação da qualidade na educação infantil}

Nesta seção, são abordados alguns parâmetros e concepções levantados por pesquisas acadêmicas, como também por documentos oficiais.

São pontos relevantes a serem considerados na avaliação da qualidade na EI: a formação de professores; a estrutura da instituição; o currículo; as práticas pedagógicas; as políticas voltadas para a primeira infância; e o trabalho pedagógico por meio da oferta de tempos, espaços, 
materialidades que promovam a ampliação de repertórios e a prática pedagógica potencializadora da aprendizagem (MEC, 2012).

Bondioli (2014) apresenta uma reflexão sobre os objetivos e os fins da avaliação. A autora não recomenda que a avaliação seja baseada em níveis do desenvolvimento e nem em testes de proficiência das crianças, não sendo aconselhável que seja realizada averiguação mediante testes e provas pontuais. "Na creche não há campos do saber, nem conteúdos específicos de aprendizagem, a contínua proposição potencializadora de experiências, verificando seu efeito sobre as capacidades e o desenvolvimento infantil” (Bondioli, 2014, p. 59).

Sousa e Pimenta (2018, p. 5) reafirmam tal concepção, apontando que

... mensurar por meio de provas externas e em larga escala não se coaduna com as finalidades da educação infantil e nem com o significado da avaliação da criança que constam nas normativas legais vigentes, as quais não prescrevem a avaliação com fins classificatórios ou seletivos.

O documento Educação infantil: Subsidios para a construção de uma sistemática nacional de avaliação (MEC, 2012) apresenta diretrizes para o estabelecimento de uma sistemática de avaliação, considerando a diversidade do contexto e as especificidades da instituição, capaz de apoiar políticas e programas educacionais. De acordo com esse documento, a avaliação deve:

- ser coerente com as finalidades e características da educação infantil;

- incluir ações coordenadas pelos diferentes níveis de governo;

- produzir informações capazes de balizar iniciativas das diversas instâncias governamentais;

- articular-se às iniciativas de avaliação institucional já em realização por redes e escolas públicas;

- ser abrangente, prevendo indicadores relativos a insumos, processos e resultados;

- considerar os determinantes intra e extra institucionais que condicionam a qualidade da educação;

- pautar-se por uma perspectiva democrática e inclusiva, não induzindo a competição em detrimento de relações compartilhadas;

- promover um processo participativo capaz de viabilizar a dimensão formativa da avaliação, estimulando diferentes atores e setores a contribuir na definição e acolhimento de parâmetros de qualidade;

- levar em conta contribuições de propostas e experiências divulgadas em âmbito nacional e internacional (MEC, 2012, pp. 18-19).

O documento pressupõe, ainda, um conjunto de aspectos que são objetos da avaliação:

1. o acesso: refere-se à oferta da educação infantil em espaços institucionais;

2. os insumos: são as condições de oferta presentes na legislação, abrangendo o financiamento, a estrutura, a garantia dos sujeitos e o serviço de apoio;

3. os processos: concernentes à gestão, ao currículo e às relações/interações presentes na instituição (MEC, 2012, p. 23).

A avaliação da educação infantil precisa prever a participação, o dialogismo, a negociação para a tomada de decisões no coletivo. Bondioli e Sávio (2013, p. 23) constataram a importância da participação como um critério necessário à qualidade, "a todos que com ela estão envolvidos de algum modo e que trabalham para explicitar e definir de maneira consensual valores, objetivos, prioridades, ideias sobre como é a instituição e sobre como deveria ou poderia ser".

Em suma, destaca-se a importância de socializar, debater e tomar decisões sobre o instrumento avaliativo com os professores e gestores que atuam diretamente na oferta dos serviços. Para Bondioli (2014, pp. 51-52), a "metodologia da avaliação deve ter caráter formativo e participativo, desde a coleta de dados". 
Festa (2019) corrobora essa ideia ao destacar a importância de promover uma avaliação da EI que de fato respeite o direito da criança, as especificidades da etapa educacional, a participação, a formação, a melhoria da qualidade e a provisão das políticas públicas.

Uma avaliação de contexto, segundo Bondioli (2014, p. 65), "para não contradizer o princípio da negociação - mesmo quando se trata de um instrumento pré-confeccionado necessita de uma escolha coerente com determinada realidade, a partir da leitura crítica desta e dos sistemas de valores".

Já quando se trata de um instrumento de avaliação externa na educação infantil, os dados são coletados e interpretados pelo avaliador externo. O contexto e os sujeitos são observados e não interferem nas decisões sobre os resultados e encaminhamentos posteriores. Para ser fidedigno, o instrumento precisa ser validado conceitualmente e por testes estatísticos.

\section{Descrição e comparação dos modelos de avaliação da educação infantil}

A pesquisa, como já apontado anteriormente, é de natureza qualitativa e se caracteriza como bibliográfica e documental. Também se configura como exploratória, por meio de buscas em sites e na literatura, não se restringindo a autores ou sistemas de ensino, pois os instrumentos de avaliação da qualidade da EI apresentam características distintas, podendo, por vezes, estar ou não atrelados às redes de ensino, ser recentes ou estar em processo de estudo, elaboração ou revisão e até mesmo estar sendo testados nos contextos.

Em 2018, Sousa e Pimenta realizaram um levantamento bibliográfico das produções que tratam da temática da avaliação da qualidade da EI. As pesquisadoras apontam a inexistência de consenso entre os autores quanto à abordagem e linearidade no tipo e na finalidade. Os modelos avaliativos são diversos e apresentam diferentes concepções, podendo referir-se à avaliação da criança, das instituições, dos professores e dos programas, por exemplo.

Pimenta (2017) apresenta uma detalhada revisão dos principais modelos de avaliação da qualidade da EI. Para além da autora, foram encontrados na literatura os trabalhos de Becchi et al. (2014), Bondioli (2009), Harms et al. (2006), Harms (2013), Marcuccio e Zanelli (2013), Moro (2018), Moro e Souza (2016), Motiejunaite et al. (2014), Myers (2011), Martínez Preciado (2010), Souza et al. (2017) e Tayler (2014).

Pimenta (2017) classifica os instrumentos de avaliação em duas categorias de análise: com foco no desenvolvimento das crianças e com foco nos ambientes, insumos e processos.

Nos modelos com foco no desenvolvimento das crianças, a avaliação atribui o conceito de qualidade com base no nível de aprendizagem dos alunos. Dentre os instrumentos que seguem essa concepção, podem-se citar: Early Years Foundation Stage; COR - Child Observation Record; ${ }^{1}$ ASQ - Ages \& Stages Questionnaires; iPIPS - Performance Indicators in Primary Schools: Baseline Assessment; e Pisa Baby - Program for International Student Assessment.

Já a segunda categoria de análise, que apresenta a avaliação da qualidade na educação infantil com foco nos ambientes, insumos e processos, avalia as condições de oferta dos serviços. Estão alinhados a essa concepção: Iters-R e Ecers-R; Isquen e AVSI; ECCP (Escala

1 O Fundo de Pesquisas Educacionais HighScope (HighScope, 2005) desenvolveu dois instrumentos de avaliação: o Child Observation Record (COR), com foco no desenvolvimento da criança; e o Program Quality Assessment (PQA), com foco em processos que abrangem vários domínios (ambiente de aprendizagem; rotina diária; interação adulto-criança; planejamento e avaliação curricular; envolvimento dos pais; qualificação e desenvolvimento de pessoal; gestão do programa). Esse programa foi um dos pesquisados para a elaboração dos Indicadores da qualidade da avaliação na educação infantil (MEC, 2009). No entanto, não será abordado no artigo por tratar-se de um amplo programa de avaliação da qualidade e devido ao não acesso ao seu conteúdo na íntegra. 
de evaluación de la calidad educativa en centros pré-escolares); NQS (National Quality Standard); Idea (Proposição de Avaliação do Instituto de Evaluación y Asesoramiento Educativo); Qualità Lúdica (Strumento per l'Analisi della Qualità Lúdica del Nido); Ervis (Elementi per Rilevare e Valutare L'Integrazione Scolastica); Spring (Strumenti per lo Sviluppo di Processi Riflessive e Indagini valutative Nei Nidi da parte dei Gruppi di lavoro educativi); e Indicadores da qualidade na educação infantil.

Os Indicadores da qualidade na educação infantil serviram de referência na construção de instrumentos avaliativos adaptados a diferentes redes e sistemas de ensino, tais como: os Indicadores de qualidade da educação infantil paulistana, que apresentam duas dimensões a mais do documento base (Secretaria Municipal de Educação, 2016); os Indicadores da qualidade na educação infantil da rede municipal de ensino de Salvador (Indique), que atribuem responsabilidades de gestão do resultado da avaliação para além da instituição (Secretaria Municipal da Educação, 2016); e os Indicadores de qualidade da educação infantil do Distrito Federal, em consonância com a Base Nacional Comum Curricular (BNCC) (Secretaria de Estado de Educação, 2019).

Outro modelo avaliativo identificado na literatura é o MELQO - Measuring Early Learning Quality and Outcomes (United Nations Educational, Scientific and Cultural Organization [Unesco], 2017), de metodologia híbrida, com foco tanto no desenvolvimento das crianças quanto nos ambientes de aprendizagem.

A seguir, os modelos baseados em ambientes, insumos e processos são descritos de modo detalhado e estruturado, com vistas a apoiar a comparação entre eles e a posterior verificação da sua adequação à realidade da RME de Florianópolis. Os modelos que envolvem a avaliação do desenvolvimento da criança não são abordados na próxima seção, pois tendem a desconsiderar a existência de infâncias distintas e a não linearidade dos ritmos de desenvolvimento infantil, conforme discutido na segunda seção.

\section{Descrição dos modelos de avaliação}

Nesta seção, os modelos de avaliação da qualidade da educação infantil, baseados em ambientes, insumos e processos, são descritos considerando-se os seguintes aspectos:

- tipo de avaliação: classificada como autoavaliação, meta-avaliação, heteroavaliação² e avaliação externa. O instrumento pode apresentar um ou combinar dois tipos ou mais;

- metodologia: o processo, as estratégias de coleta de dados, presença de avaliadores;

- tempo: período destinado à observação e coleta de dados;

- indicadores: são variáveis operativas que verificam o nível de qualidade, valoração;

- participação: promove a reflexão dos diferentes segmentos (profissionais e famílias) sobre experiências pedagógicas e seus valores educativos;

- resultados: relacionados ao objetivo, contemplando os parâmetros pretendidos;

- pós-resultados: encaminhamentos posteriores ao resultado, plano de ação e melhorias;

- viabilidade: apresenta pertinência contextual, indicadores alcançáveis, alinhados às diretrizes e à especificidade da EI e viáveis financeiramente; qualifica os avaliadores;

- validade e confiabilidade: têm consistência interna; capacidade de representar e dar valor à realidade avaliada com abrangência e articulação aos parâmetros de qualidade;

- maturidade: refere-se ao nível de eficiência do instrumento na potencialização de melhorias e na sua capacidade de adaptação em diferentes contextos e tempos históricos.

2 Autoavaliação: a instituição faz uma avaliação interna a partir dos indicadores de um instrumento avaliativo. Meta-avaliação: o objetivo é fazer uma avaliação da avaliação, ou seja, uma avaliação dos resultados. Heteroavaliação: avaliação feita por uma ou mais pessoas ou entidades que não estão sendo avaliadas no momento (Gariboldi \& Maselli, 2018). 
Partindo das informações do referencial bibliográfico, o Quadro 1 traz a descrição dos instrumentos a fim de conhecer as especificidades de cada modelo.

\section{QUADRO 1}

\section{Descrição dos modelos de avaliação da educação infantil}

\begin{tabular}{|c|c|c|}
\hline \multicolumn{3}{|c|}{ ITERS-R e ECERS-R - Estados Unidos } \\
\hline Tipo & \multicolumn{2}{|c|}{ Avaliação externa } \\
\hline Metodologia & \multicolumn{2}{|c|}{$\begin{array}{l}\text { Um avaliador externo observa os espaços e ambientes, identifica e atribui conceito de qualidade ao } \\
\text { longo dos indicadores. }\end{array}$} \\
\hline Indicadores & $\begin{array}{l}\text { Iters-R } \\
\text { Avalia o espaço e mobiliário; rotinas de } \\
\text { cuidado pessoal; falar e compreender; } \\
\text { atividades; interação; estrutura do } \\
\text { programa, pais e equipe, com } 39 \text { itens } \\
\text { detalhados em } 455 \text { indicadores. }\end{array}$ & $\begin{array}{c}\text { Ecers-R } \\
\text { Avalia o espaço e mobiliário; rotinas de cuidado } \\
\text { pessoal, linguagem e raciocínio; atividades; interação, } \\
\text { estrutura do programa; pais e equipe, com } 43 \text { itens e } 470 \\
\text { indicadores. }\end{array}$ \\
\hline Tempo & \multicolumn{2}{|c|}{ Um dia, período de 3,5 horas de observação. } \\
\hline Participação & \multicolumn{2}{|c|}{$\begin{array}{c}\text { Não apresentam característica participativa, apenas são consultados os pais e a equipe quando o } \\
\text { avaliador externo não identifica os indicadores no ambiente. }\end{array}$} \\
\hline Resultados & \multicolumn{2}{|c|}{$\begin{array}{c}\text { Os dados e informações são coletados e mensurados, sendo atribuído um valor pelo avaliador que não } \\
\text { discute os resultados da avaliação. }\end{array}$} \\
\hline Pós-resultados & \multicolumn{2}{|c|}{ O avaliador externo faz os indicativos sobre pontos a serem melhorados e estratégias de ação. } \\
\hline Viabilidade & \multicolumn{2}{|c|}{ Viáveis pela facilidade de acesso e pouco tempo na preparação dos avaliadores externos. } \\
\hline $\begin{array}{l}\text { Validade e } \\
\text { confiabilidade }\end{array}$ & \multicolumn{2}{|c|}{$\begin{array}{l}\text { Válidos e confiáveis por serem abrangentes com parâmetros comuns da educação infantil, facilitando } \\
\text { o mapeamento da qualidade em realidades sociais e educacionais bem diversas. }\end{array}$} \\
\hline Maturidade & \multicolumn{2}{|c|}{$\begin{array}{l}\text { Grau de maturidade estabelecido por terem sido aplicados em tempos históricos distintos e em } \\
\text { diferentes contextos dos Estados Unidos e mais tarde em países dos cinco continentes. }\end{array}$} \\
\hline \multicolumn{3}{|c|}{ Fonte: Harms et al. (2006), Harms (2013) e Pimenta (2017). } \\
\hline \multicolumn{3}{|c|}{ ISQUEN e AVSI - Itália } \\
\hline Tipo & \multicolumn{2}{|c|}{ Meta-avaliação e autoavaliação } \\
\hline Metodologia & \multicolumn{2}{|c|}{$\begin{array}{c}\text { Constituídos por um avaliador externo, avaliadores internos (entre eles, um articulador) e outros } \\
\text { segmentos da instituição (famílias e profissionais). Apresentam indicadores predefinidos e promovem } \\
\text { o debate entre os participantes de modo processual e formativo. }\end{array}$} \\
\hline Indicadores & $\begin{array}{l}\text { Isquen } \\
\text { Os sujeitos, os contextos e práticas, os } \\
\text { saberes do fazer, as garantias. }\end{array}$ & $\begin{array}{l}\text { AVSI } \\
\text { Experiência educativa, as atividades profissionais, os } \\
\text { adultos e suas relações, as garantias, a estrutura. }\end{array}$ \\
\hline Tempo & \multicolumn{2}{|c|}{ Varia de uma instituição para outra. Não é descrito o tempo de aplicação, nem a regularidade. } \\
\hline Participação & \multicolumn{2}{|c|}{ De natureza negociada e dialógica, preveem a participação de todos os segmentos. } \\
\hline Resultados & \multicolumn{2}{|c|}{$\begin{array}{l}\text { Os itens com discordância são socializados a fim de refletir e encontrar o consenso a partir da } \\
\text { concepção, dos valores daquela realidade e dos princípios da educação infantil. }\end{array}$} \\
\hline Pós-resultados & \multicolumn{2}{|c|}{ Elaboração de um plano de ação coletivo, tornando todos coadjuvantes das melhorias. } \\
\hline Viabilidade & \multicolumn{2}{|c|}{$\begin{array}{c}\text { Por serem processuais e pela interação do avaliador externo, enquanto articulador do instrumento, } \\
\text { exigem maior qualificação e tempo. }\end{array}$} \\
\hline $\begin{array}{l}\text { Validade e } \\
\text { confiabilidade }\end{array}$ & \multicolumn{2}{|c|}{$\begin{array}{l}\text { Válidos e confiáveis. Apresentam consistência interna, a avaliação foi construída a partir da adaptação } \\
\text { da Iters e Ecers, preveem a adaptação à realidade contextual. }\end{array}$} \\
\hline Maturidade & \multicolumn{2}{|c|}{$\begin{array}{l}\text { Grau de maturidade estabelecido, também aplicados em outros países. No entanto, por avaliarem o } \\
\text { contexto, necessitam de adaptação à realidade observada. }\end{array}$} \\
\hline
\end{tabular}




\begin{tabular}{|c|c|}
\hline \multicolumn{2}{|r|}{ ECCP - México } \\
\hline Тіро & Avaliação externa \\
\hline Metodologia & $\begin{array}{l}\text { Avaliação de programas realizada por avaliadores externos. O instrumento combina observação dos } \\
\text { espaços e entrevistas com diretores e professores relacionados à instituição e à sala de aula. }\end{array}$ \\
\hline Indicadores & $\begin{array}{l}\text { Estabelecimento, recursos, processo educacional, gestão educacional, relação com família e } \\
\text { comunidade, sala de aula, recursos. }\end{array}$ \\
\hline Tempo & $\begin{array}{l}\text { Avaliadores externos: visitas no início e final do ano letivo. Dois dias com entrevistas, famílias e } \\
\text { crianças. Três visitas: uma para discussão sobre os resultados, verificando a validade, e duas para } \\
\text { elaborar ações de melhoria com professores e diretores. E, por último, três visitas para verificar o } \\
\text { desenvolvimento das ações. }\end{array}$ \\
\hline Participação & $\begin{array}{l}\text { O instrumento combina observação e entrevistas, mas o parecer sobre a qualidade é realizado pelos } \\
\text { avaliadores externos. }\end{array}$ \\
\hline Resultados & $\begin{array}{l}\text { Constataram-se melhorias na qualidade da infraestrutura, materiais, gestão, saúde, identidade } \\
\text { institucional, relação creche-família. }\end{array}$ \\
\hline Pós-resultados & $\begin{array}{c}\text { Feedback da avaliação é a base para a continuidade dos currículos e reorientar a formação de } \\
\text { professores e supervisão. }\end{array}$ \\
\hline Viabilidade & $\begin{array}{l}\text { Instrumento construído pelo sistema educacional mexicano entre } 2003 \text { e } 2007 \text {, buscando adequar-se } \\
\text { às diretrizes (princípios gerais da educação infantil); viável para aquele contexto. }\end{array}$ \\
\hline $\begin{array}{l}\text { Validade e } \\
\text { confiabilidade }\end{array}$ & $\begin{array}{l}\text { Não descreve. Instrumento em período de teste de uma avaliação padrão para um contexto diverso e } \\
\text { multicultural, que coincidiu com a ampliação da oferta de vagas. }\end{array}$ \\
\hline Maturidade & $\begin{array}{l}\text { Nível de maturidade não definido. Foi reformulado cinco vezes, incluindo novas dimensões: saúde, } \\
\text { clima, crianças com deficiência. }\end{array}$ \\
\hline \multicolumn{2}{|c|}{ Fonte: Myers (2011) e Martínez Preciado (2010). } \\
\hline \multicolumn{2}{|r|}{ NQS - Austrália } \\
\hline Tipo & Avaliação externa \\
\hline Metodologia & $\begin{array}{l}\text { Avaliador externo avalia a qualidade dos serviços. Visitas de agente autorizado para observar os } \\
\text { espaços, questionar, analisar documentos, verificar a aplicação do plano. }\end{array}$ \\
\hline Indicadores & $\begin{array}{l}\text { Programa educacional e prática; saúde e segurança das crianças; ambiente físico; arranjos de pessoal; } \\
\text { relações com as crianças; parcerias de colaboração com as famílias e comunidades; liderança e gestão } \\
\text { dos serviços. }\end{array}$ \\
\hline Tempo & Não é descrito o tempo de aplicação. \\
\hline Participação & $\begin{array}{l}\text { Participação limitada aos profissionais e famílias. Os avaliadores externos têm grande influência na } \\
\text { decisão sobre o resultado. }\end{array}$ \\
\hline Resultados & $\begin{array}{l}\text { Legislação prevê a divulgação dos resultados (avaliação reguladora). Devem ser alcançados níveis de } \\
\text { qualidade para o credenciamento das instituições. }\end{array}$ \\
\hline Pós-resultados & $\begin{array}{l}\text { Penalidades são previstas em lei para as instituições que não estiverem atendendo aos níveis de } \\
\text { qualidade estabelecidos no plano de melhorias. Sujeito à responsabilização dos profissionais, das } \\
\text { instituições e suspensões dos serviços. }\end{array}$ \\
\hline Viabilidade & Viável. Por ser instituído, articulado e garantido por lei, já prevê recursos para realização. \\
\hline $\begin{array}{l}\text { Validade e } \\
\text { confiabilidade }\end{array}$ & $\begin{array}{l}\text { Reconhece a existência de contextos multiculturais, apresenta coerência com as diretrizes nacionais, } \\
\text { tornando o instrumento confiável e válido, mas abre precedentes para ranqueamento das instituições. }\end{array}$ \\
\hline Maturidade & Tem nível de maturação consistente. É sistêmica, abrangente, padronizada nacionalmente. \\
\hline \multicolumn{2}{|c|}{ Fonte: Tayler (2014) e Pimenta (2017). } \\
\hline \multicolumn{2}{|r|}{ IDEA - Espanha } \\
\hline Tipo & Autoavaliação \\
\hline Metodologia & $\begin{array}{l}\text { Por adesão voluntária da instituição. Composta por etapas: } \\
\text { a) coleta de informação: entrevistas, revisão de documentos, observação, aplicação de questionários; } \\
\text { b) valoração: elaboração de informes e destaque de pontos fortes e fracos; } \\
\text { c) tomada de decisões: propostas de melhoria. }\end{array}$ \\
\hline Indicadores & $\begin{array}{c}\text { A formação docente, a quantidade de crianças por turma, a estrutura física em relação à saúde } \\
\text { e segurança (em edifícios, instalações, equipamentos interiores e exteriores), o currículo e as } \\
\text { aprendizagens e a prática educativa. }\end{array}$ \\
\hline
\end{tabular}




\begin{tabular}{|c|c|}
\hline Tempo & Não descreve o tempo de aplicação do instrumento. \\
\hline Participação & $\begin{array}{l}\text { Participação condicionada ao questionário de valoração aplicado às famílias e aos professores. Os } \\
\text { avaliadores externos definem o nível de qualidade. }\end{array}$ \\
\hline Resultados & Destaques dos pontos fracos e fortes da instituição. \\
\hline Pós-resultados & Há a proposição de melhorias, mas não descreve como é feito. \\
\hline Viabilidade & Não descreve dificuldade ou êxito na aplicação. \\
\hline $\begin{array}{l}\text { Validade e } \\
\text { confiabilidade }\end{array}$ & $\begin{array}{c}\text { Como é uma autoavaliação por adesão da instituição, as informações são insuficientes quanto à } \\
\text { eficiência, validade e confiabilidade. }\end{array}$ \\
\hline Maturidade & Não descreve o nível de maturação. \\
\hline \multicolumn{2}{|c|}{ Fonte: Motiejunaite et al. (2014) e Pimenta (2017). } \\
\hline \multicolumn{2}{|r|}{ QUALITÀ LÚDICA - Itália } \\
\hline Tipo & Autoavaliação \\
\hline Metodologia & $\begin{array}{l}\text { Observação sistemática dos espaços e coleta de dados dos avaliadores sobre a ampliação do } \\
\text { repertório de brincadeira e dos comportamentos lúdicos e competências das crianças. }\end{array}$ \\
\hline Indicadores & $\begin{array}{l}\text { Projeto educativo; espaço (na sala, nas áreas comuns internas e externas); materiais (na sala, nas áreas } \\
\text { comuns internas e externas); tempo; formação do grupo; adulto e jogo. }\end{array}$ \\
\hline Tempo & Não é descrito o tempo de aplicação. \\
\hline Participação & Promove a participação na perspectiva da avaliação de contexto. \\
\hline Resultados & $\begin{array}{l}\text { Os dados levantados são debatidos com o avaliador externo e os segmentos da comunidade educativa } \\
\text { para chegar a um consenso. }\end{array}$ \\
\hline Pós-resultados & Não descreve. \\
\hline Viabilidade & $\begin{array}{l}\text { Não descreve, requer conhecimento sobre desenvolvimento infantil e a brincadeira, exigindo maior } \\
\text { qualificação e mediação do avaliador externo. }\end{array}$ \\
\hline $\begin{array}{l}\text { Validade e } \\
\text { confiabilidade }\end{array}$ & $\begin{array}{l}\text { Avaliação subjetiva, pois depende da interpretação dos avaliadores. O instrumento não apresenta } \\
\text { informações suficientes sobre o grau de confiabilidade. }\end{array}$ \\
\hline Maturidade & Não é descrito o nível de maturação. Encontra-se ainda como projeto piloto, de pesquisa. \\
\hline \multicolumn{2}{|c|}{ Fonte: Moro e Souza (2016). } \\
\hline \multicolumn{2}{|r|}{ ERVIS } \\
\hline Tipo & Autoavaliação - educação inclusiva \\
\hline Metodologia & $\begin{array}{l}\text { Composto por cinco áreas avaliadas, subdivididas em } 41 \text { itens, observação dos espaços realizada pelos } \\
\text { profissionais, de preferência com formação em educação inclusiva, mas as melhorias são destinadas a } \\
\text { todas as crianças. }\end{array}$ \\
\hline Indicadores & Estrutura; formação; atividades profissionais; relação entre adultos; processo de integração. \\
\hline Tempo & Não descreve o tempo de aplicação. \\
\hline Participação & Participativa, dialógica com os profissionais da instituição. \\
\hline Resultados & $\begin{array}{l}\text { Dados visam a identificar a presença ou ausência de fatores significativos à educação de crianças } \\
\text { com deficiência. }\end{array}$ \\
\hline Pós-resultados & $\begin{array}{l}\text { Com os resultados se elabora um projeto específico, com a participação dos professores para } \\
\text { propor, implementar, avaliar e modificar itinerários personalizados destinados a ensinar as } \\
\text { crianças "deficientes". }\end{array}$ \\
\hline Viabilidade & Contextual, demanda formação específica em educação especial. \\
\hline $\begin{array}{l}\text { Validade e } \\
\text { confiabilidade }\end{array}$ & $\begin{array}{l}\text { Não apresenta informações suficientes sobre o grau de confiabilidade, pois depende da interpretação } \\
\text { dos avaliadores, assim como do domínio dos processos inclusivos. }\end{array}$ \\
\hline Maturidade & Não foi possível identificar o nível de maturação. \\
\hline te: Bondioli & ) e Pimenta (2017). \\
\hline
\end{tabular}




\begin{tabular}{|c|c|}
\hline \multicolumn{2}{|r|}{ SPRING - Itália } \\
\hline Tipo & Autoavaliação e heteroavaliação \\
\hline Metodologia & $\begin{array}{l}\text { O avaliador externo faz o contato inicial, reuniões, entrevistas e observações dos espaços e práticas, } \\
\text { elaboração do relatório final sobre os dados levantados, debatidos e analisados para a devolutiva } \\
\text { à instituição. Com dimensões que se relacionam entre si, servem tanto para autoavaliação quanto } \\
\text { heteroavaliação. Não são utilizados dados quantificáveis, sendo totalmente descritiva, requer tempo } \\
\text { pela subjetividade, inclusive para consulta aos resultados. }\end{array}$ \\
\hline Indicadores & $\begin{array}{l}\text { Organização do contexto educativo; funcionamento do grupo de trabalho; relações da instituição com } \\
\text { as famílias e a comunidade; processos de avaliação. }\end{array}$ \\
\hline Tempo & Em média seis meses. A reaplicação depende da evolução no plano de melhorias. \\
\hline Participação & $\begin{array}{l}\text { Prevê a participação dos diferentes segmentos da instituição, formativa, dialógica, de debate e } \\
\text { confronto, inclusive de concepções pedagógicas para qualidade negociada. }\end{array}$ \\
\hline Resultados & Elaboração de plano de melhorias. \\
\hline Pós-resultados & Colocar em prática o plano de melhorias com as mudanças e continuidades indicadas. \\
\hline Viabilidade & $\begin{array}{l}\text { Viável, em uma perspectiva sistêmica, com estrutura humana qualificada, de conhecimento específico } \\
\text { sobre o instrumento e concepção pedagógica para dar suporte à instituição. }\end{array}$ \\
\hline $\begin{array}{l}\text { Validade e } \\
\text { confiabilidade }\end{array}$ & $\begin{array}{l}\text { Dispõe de consistência interna, pois apresenta análise do contexto sob o olhar de diferentes } \\
\text { segmentos, propiciando a validade e confiabilidade. }\end{array}$ \\
\hline Maturidade & Não descreve. Instrumento criado recentemente. \\
\hline \multicolumn{2}{|c|}{ Fonte: Marcuccio e Zanelli (2013) e Moro (2018). } \\
\hline \multicolumn{2}{|r|}{ INDICADORES DA QUALIDADE DA EDUCAÇÃO INFANTIL - Brasil } \\
\hline Tipo & Autoavaliação \\
\hline Metodologia & $\begin{array}{l}\text { Os participantes são divididos em sete equipes, uma para cada dimensão, contando com coordenador } \\
\text { e relator. O nível de qualidade é baseado nas cores do semáforo. Ao concluir essa etapa, os subgrupos } \\
\text { retornam à plenária e cada dimensão é retomada, com o intuito de negociar e chegar a um consenso } \\
\text { quanto ao resultado e elaborar o plano de melhorias. }\end{array}$ \\
\hline Indicadores & $\begin{array}{l}\text { Planejamento institucional; multiplicidade de experiências e linguagens; interações; promoção da } \\
\text { saúde; espaços, materiais e mobiliários; formação e condições de trabalho das professoras e demais } \\
\text { profissionais; cooperação e troca com as famílias e participação na rede de proteção social. }\end{array}$ \\
\hline Tempo & Um dia. \\
\hline Participação & São fundamentais a participação e o posicionamento dos diferentes segmentos da instituição. \\
\hline Resultados & $\begin{array}{l}\text { Identificar os pontos fortes e fracos da instituição para elaboração do plano de ação, constando: } \\
\text { dimensão, indicador, problemas, ações, responsáveis e prazos. }\end{array}$ \\
\hline Pós-resultados & $\begin{array}{l}\text { Não propõe o acompanhamento do plano de ação por parte de todos os segmentos, nem propõe } \\
\text { atribuições à Secretaria Municipal de Educação e/ou políticas públicas. }\end{array}$ \\
\hline Viabilidade & Acessível e de fácil aplicação. \\
\hline $\begin{array}{l}\text { Validade e } \\
\text { confiabilidade }\end{array}$ & $\begin{array}{l}\text { Por não ser formativa, interfere na validade e confiabilidade. Depende da concepção e do } \\
\text { reconhecimento da comunidade educativa para identificar a qualidade dos serviços. }\end{array}$ \\
\hline Maturidade & Não descreve nível de maturidade, mas pode ser aplicada em diferentes tempos e contextos. \\
\hline
\end{tabular}

Fonte: Elaboração própria.

\section{Análise comparada dos modelos de avaliação da qualidade na educação infantil}

Partindo do levantamento das características dos modelos de avaliação estrangeiros e nacionais, pretende-se, nesta seção, compará-los a partir de critérios que buscam refletir sobre os parâmetros a serem considerados em um modelo avaliativo para que estejam em consonância com os princípios da educação infantil. Esses critérios de comparação foram definidos com base no estudo dos parâmetros de qualidade, apresentados na segunda seção. 
O Quadro 2 mostra os resultados da análise comparada entre os modelos de avaliação da educação infantil segundo os critérios estabelecidos.

\section{QUADRO 2}

Análise comparada entre os modelos de avaliação da educação infantil

\begin{tabular}{|c|c|c|c|c|c|c|c|c|c|}
\hline Modelo de avaliação & 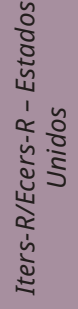 & 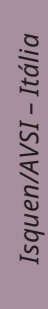 & 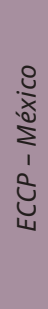 & 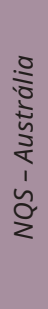 & $\begin{array}{l}\text { g } \\
\frac{1}{5} \\
\delta \\
\frac{0}{4} \\
1 \\
\delta \\
\frac{d}{2}\end{array}$ & 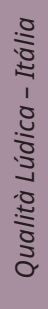 & 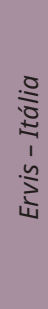 & $\begin{array}{l}0 \\
: 0 \\
0 \\
1 \\
1 \\
0 \\
\vdots \\
\vdots \\
0 \\
\text { n. }\end{array}$ & 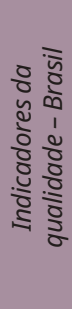 \\
\hline $\begin{array}{l}\text { 1. Em consonância com as diretrizes curriculares da } \\
\text { educação infantil (EI) brasileira }\end{array}$ & @ & @ & @ & @ & @ & @ & @ & @ & $S$ \\
\hline $\begin{array}{l}\text { 2. Indica o padrão de qualidade, conforme os princípios e } \\
\text { parâmetros da EI brasileira }\end{array}$ & @ & @ & @ & @ & @ & $!$ & $!$ & @ & S \\
\hline $\begin{array}{l}\text { 3. Considera como parâmetro de qualidade os valores e } \\
\text { finalidades do contexto }\end{array}$ & * & $S$ & @ & @ & $!$ & $S$ & $S$ & $S$ & $\mathrm{~s}$ \\
\hline $\begin{array}{l}\text { 4. Promove a participação de todos os segmentos da } \\
\text { comunidade educativa }\end{array}$ & * & $S$ & * & N & * & $S$ & $S$ & $S$ & S \\
\hline $\begin{array}{l}\text { 5. A metodologia prevê avaliador externo e avaliador } \\
\text { interno }\end{array}$ & N & $S$ & N & N & $\mathrm{N}$ & $S$ & S & $S$ & N \\
\hline 6. Promove avaliação como processo formativo & $\mathrm{N}$ & $\mathrm{s}$ & $\mathrm{N}$ & $\mathrm{N}$ & $\mathrm{N}$ & $\mathrm{s}$ & $\mathrm{s}$ & $\mathrm{s}$ & * \\
\hline $\begin{array}{l}\text { 7. Por ser dialógica, há consenso no resultado para a } \\
\text { validação do constructo }\end{array}$ & N & $S$ & N & N & $\mathrm{N}$ & $\mathrm{s}$ & S & $S$ & S \\
\hline $\begin{array}{l}\text { 8. Prevê a elaboração coletiva do plano de melhorias e a } \\
\text { gestão das ações }\end{array}$ & N & $S$ & $\mathrm{~N}$ & N & $\mathrm{N}$ & $S$ & $S$ & $S$ & $S$ \\
\hline $\begin{array}{l}\text { 9. Permite o acompanhamento do plano de melhorias pela } \\
\text { comunidade educativa }\end{array}$ & N & $S$ & @ & @ & @ & $!$ & $!$ & $S$ & S \\
\hline $\begin{array}{l}\text { 10. Prevê a intersetorialidade por meio de uma rede de } \\
\text { apoio externa à instituição }\end{array}$ & $\mathrm{N}$ & * & N & N & $!$ & $!$ & $\mathrm{N}$ & N & * \\
\hline $\begin{array}{l}\text { 11. Apresenta nível de validade, confiabilidade e } \\
\text { maturidade consolidado }\end{array}$ & $S$ & $S$ & * & S & $!$ & $!$ & $!$ & $!$ & * \\
\hline
\end{tabular}

Fonte: Elaboração própria.

Pode-se observar que, dos modelos analisados, apenas aquele referente aos Indicadores da qualidade na educação infantil está em completa consonância com as diretrizes curriculares da EI brasileira, sendo também o único que indica um padrão de qualidade em conformidade com os princípios e parâmetros da EI nacional. Sua definição foi assim descrita:

. . . adentra a discussão do conceito de qualidade, flexível, negociável em face das diferentes perspectivas que se adotam: socialmente construído; dependente do contexto; baseado em direitos, necessidades, demandas, conhecimentos e possibilidades. Indicam-se como passíveis de avaliação: as políticas para a Educação Infantil, sua implementação e acompanhamento; as propostas pedagógicas das instituições de Educação Infantil; a relação estabelecida com as famílias das crianças; a formação regular e continuada dos professores e demais profissionais; a infraestrutura necessária ao funcionamento dessas instituições. Em relação à avaliação das crianças, reitera que ela não deve implicar a retenção das crianças na Educação Infantil. (MEC, 2015, pp. 16-17). 
Juntamente com os Indicadores da qualidade na educação infantil, os instrumentos de avaliação italianos Isquen/AVSI, Qualità Lúdica, Ervis e Spring, pela capacidade de adaptação ao contexto, são os que mais contemplam itens favoráveis aos critérios estabelecidos.

Quanto aos critérios sobre promoção, participação dos diferentes segmentos da instituição, consideração da diversidade contextual na avaliação, promoção da formação durante o processo avaliativo e disposição de avaliador externo e avaliador interno para assegurar o consenso no resultado da avaliação, nota-se que os modelos italianos Isquen/AVSI, Qualità Lúdica, Ervis e Spring conseguem contemplá-los. Os demais instrumentos não atendem satisfatoriamente a tais critérios, ou, então, preveem participação e contextualização, mas não as efetivam quando a avaliação é colocada em prática. Apenas os instrumentos italianos promovem avaliação formativa. Os Indicadores da qualidade na educação infantil pressupõem a participação e dialogam consensualmente quanto ao resultado para a validação da avaliação, e promovem a elaboração de um plano de melhorias e o acompanhamento na gestão das ações.

Ainda, os modelos italianos Isquen/AVSI, Qualità Lúdica, Ervis e Spring e os Indicadores da qualidade na educação infantil são os que apresentam maior possibilidade de adaptação, ou já estão em consonância com os parâmetros de qualidade no quesito relacionado aos valores e às finalidades do contexto. A ECCP e a NQS necessitam de adaptação em sua estrutura, correndo o risco de alterar sua concepção e essência, pois reconhecem a importância de contemplar a diversidade de contextos e populações.

Alguns modelos, como o Iters-R/Ecers-R, ECCP, Idea e NQS, não apresentam característica de uma avaliação de contexto, formativa e participativa, conforme discutido anteriormente, não sendo indicados para os contextos brasileiro e florianopolitano sem mudanças na sua estrutura metodológica, nos fins e objetivos da avaliação.

Há os modelos em que a avaliação é hierárquica (Iters-R/Ecers-R, ECCP, NQS e Idea), centrada na figura do avaliador externo, que atribui conceito de qualidade conforme suas percepções, baseando-se no resultado observado no indicador em questão para a definição das ações de melhorias da qualidade.

Um dos critérios de qualidade, debatido neste estudo, trata da participação como requisito importante para um processo avaliativo democrático e dialógico. Desse modo, os modelos supracitados têm como característica não considerar a participação da comunidade educativa, tornando-os apenas objetos e não sujeitos da avaliação. Diante disso, o instrumento torna-se vulnerável no que se refere às especificidades da educação infantil, sem promover a participação dos diferentes atores (profissionais, gestores, famílias) que desenvolvem o trabalho, sendo que a participação é intrínseca à qualidade.

Com relação à elaboração de um plano de melhorias e acompanhamento, pode-se observar que os modelos italianos Isquen/AVSI e Spring também contemplam esse critério. Mesmo que usem outra nomenclatura, há o consenso quanto à necessidade de planejar, gerenciar e acompanhar a continuidade da avaliação. Nesse sentido, a avaliação de contexto não apresenta uma linearidade na temporalidade de (re)aplicação da avaliação nas instituições, devido às suas singularidades identitárias e tempos distintos na implementação das melhorias.

A intersetorialidade é um aspecto crítico de todos os modelos de avaliação. Alguns até a preveem, como é o caso da avaliação de contexto Isquen/AVSI, Spring e os Indicadores da qualidade da EI, mas não conseguem ter uma rede de apoio externa à instituição de setores como da saúde e da assistência social, que viabilize o debate sobre a qualidade e a superação de problemas sociais que afetam a vida das crianças e, consequentemente, a educação infantil. Essa ação conjunta poderia proporcionar a garantia do direito da criança na sua integralidade. Posto isso, é imprescindível a indissociabilidade entre a política de avaliação, capaz de assegurar 
que o instrumento avaliativo apresente em sua metodologia os indicadores relacionados à intersetorialidade, e a garantia de fato dessa condição.

Para finalizar esta parte, evidencia-se que o nível de maturação se estabelece quando o conhecimento técnico e a possibilidade de operacionalização validam a continuidade do processo avaliativo, permitindo sua aplicação em outros contextos. Nesse viés, a validação e a confiabilidade estão associadas ao grau de maturação do instrumento, já consolidado nos modelos Iters-R/Ecers-R, Isquen/AVSI e NQS. Já os Indicadores da qualidade na educação infantil, ECCP e Spring preveem, mas não garantem, a confiabilidade, em razão da descontinuidade e mudança de metodologia da avaliação, que interferem no nível de maturação do instrumento.

\section{Discussão dos resultados}

Conforme observado na análise comparada dos modelos de avaliação, os Indicadores da qualidade na educação infantil (MEC, 2009) estão alinhados aos parâmetros da legislação educacional e atendem à maioria dos aspectos desejáveis em um instrumento de avaliação da qualidade da educação infantil, colocando o modelo avaliativo em maior consonância com a RME de Florianópolis, embora necessite ser ancorado por uma política de avaliação capaz de garantir uma avaliação formativa e prever uma política de intersetorialidade.

Desde a década de 1990, na construção de diretrizes e orientações, a RME de Florianópolis apresenta consonância com a legislação e as concepções anunciadas nos documentos nacionais, em especial com as Diretrizes Curriculares Nacionais para a Educação Infantil (MEC, 2010).

Os Indicadores da qualidade na educação infantil dialogam com os documentos curriculares $^{3}$ da RME de Florianópolis ao promoverem a participação e o debate, considerando as especificidades, a identidade e a cultura do contexto histórico, social e econômico; preveem o diálogo em torno da oferta dos serviços com base nas condições estruturais, pedagógicas e relacionais, enquanto promotoras da ampliação de conhecimentos científicos por meio do acesso às condições materiais e pedagógicas, contribuindo, assim, para aprendizagem e desenvolvimento das crianças. Esse instrumento tem a capacidade de promover uma avaliação viável, válida e confiável para o mapeamento e a gestão dos resultados.

As avaliações desenvolvidas e adaptadas nos diferentes países mostram a necessidade de constância no mapeamento da qualidade. A Itália apresenta uma trajetória avaliativa que se iniciou na adaptação de instrumentos norte-a mericanos, até a construção de sua própria avaliação. O sistema de ensino italiano não estabelece um único modelo nacional, mas tem como critério comum contemplar os elementos da realidade local, bem como a participação, tendo em conta a diversidade contextual das instituições.

Os modelos de avaliação italianos também se aproximam da proposta pedagógica da RME de Florianópolis, pela influência da pedagogia italiana na sua base curricular e documentos orientadores e na formação dos profissionais e, além disso, estão relacionados aos valores e finalidades do contexto. Essa constatação levanta a segunda possibilidade avaliativa para a realidade de Florianópolis: a avaliação de contexto. Dentre os modelos italianos estudados, o Spring é um dos que melhor se adequariam à aplicação no contexto florianopolitano, porém,

3 Diretrizes Educacionais Pedagógicas para a Educação Infantil (2010), Orientações Curriculares para a Educação Infantil da RME de Florianópolis (2012), Currículo da Educação Infantil da RME de Florianópolis (2015), Diretrizes Curriculares para a Educação Básica da Rede Municipal de Ensino de Florianópolis (2015), Matriz Curricular para Educação das Relações ÉtnicoRaciais na Educação Básica (2016), A Educação Física na Educação Infantil da Rede Municipal de Ensino de Florianópolis (2016). http://www.pmf.sc.gov.br/entidades/educa/index.php?cms=legislacao++leis+e+orientacoes+++dei 
precisa de algumas adaptações, especialmente no quesito análise dos resultados, os quais, por serem descritivos, dificultam a otimização do acesso e da gestão da avaliação.

A exemplo da Itália, constata-se a necessidade de os gestores e o corpo docente conhecerem os diferentes instrumentos de avaliação, em um processo de pesquisa-ação, para, então, fazerem escolhas, adaptarem ao contexto e, até mesmo, elaborarem um instrumento de avaliação baseado nos princípios e diretrizes da EI. Entretanto, a RME de Florianópolis, mesmo sem dispor de uma política avaliativa instituída, encontra-se em processo de construção de seu próprio instrumento, avaliação de contexto.

A iniciativa está vinculada aos processos de formação continuada sobre sua base curricular, sendo este um espaço profícuo de debate formativo e democrático em torno dos próprios resultados. Desse modo, tal atitude não se configura apenas como campo de estudo proposto pelas experiências avaliativas da qualidade da EI, mas há também a possibilidade de construção do próprio instrumento, a partir do cotejamento dos documentos curriculares.

O GT para a elaboração do instrumento de avaliação foi constituído, em 2018, por profissionais da educação infantil, que representam as cinco regiões que compõem geograficamente o município, tendo a contribuição de oito assessoras do Núcleo de Formação, Pesquisa e Assessoramento da Educação Infantil (Nufpaei), com a consultoria de Catarina Moro e Angela Scalabrin Coutinho, da UFPR. Algumas ações foram realizadas nesse percurso. Inicialmente o GT participou de encontros relacionados a: estudos ${ }^{4}$ teóricos e conceituais sobre avaliação da educação infantil; qualidade e participação; avaliação de contexto; indicadores de qualidade; dimensões e análises dos documentos curriculares da educação infantil da RME de Florianópolis e de alguns instrumentos de avaliação nacionais e internacionais. Em setembro de 2018, organizado pelo Nufpaei, aconteceu o primeiro seminário Avaliação da e na Educação Infantil. Foi o início de um compartilhamento com representantes das unidades educativas sobre o processo de constituição do instrumento de avaliação da qualidade.

A partir das escolhas metodológicas e de concepções mapeadas e desenvolvidas pelo GT na construção da avaliação de contexto, foi composta a proposta para as dimensões do instrumento, quais sejam: relações educativo-pedagógicas; estratégias da ação pedagógica; relações; gestão e aportes fundamentais. Simultaneamente a essa construção, em 2019, foram formados grupos de estudos - de caráter formativo participativo e representativo -, nas regióes do município, para o compartilhamento do percurso de escrita do instrumento. Por sua vez, os representantes de cada unidade educativa tinham a tarefa de socializar em seu local de trabalho as informações apresentadas nas regionais. Também cabia ao GT expor, nos encontros formativos, o debate e as demandas apresentadas nas regionais.

O segundo seminário - Avaliação de Contexto na Educação Infantil - aconteceu em novembro de 2019, com o objetivo de aprofundar a perspectiva teórica, bem como conhecer e dialogar com os processos avaliativos da avaliação de contexto na EI, desenvolvidos na região de Romagna na Itália, coordenados por Antônio Gariboldi. Nesse seminário foram apresentadas a escolha metodológica do instrumento e as principais dimensões que o compõem.

Em 2020 e 2021, o processo de elaboração do instrumento avaliativo teve continuidade e vem se constituindo à medida que o GT vai delineando os princípios metodológicos, a escrita e as estratégias de sua operacionalização. ${ }^{5}$ Atualmente, a primeira dimensão encontra-se em processo

4 Informativo realizado pelo Nufpaei referente à Contextualização da avaliação da educação infantil na rede municipal de ensino de Florianópolis.

5 Opercurso deelaboração do do instrumento deavaliação da RME de Florianópolis, bem como parte das referências consultadas na sua construção, encontra-se disponível em: https://sites.google.com/sme.pmf.sc.gov.br/portaleducacionaledinfantil/ avalia\%C3\%A7\%C3\%A3o-de-contexto 
de finalização. Desse modo, a previsão temporal de sua conclusão depende de inúmeras variáveis, dentre as quais a viabilidade de aplicação do instrumento nas unidades educativas, diante do atual cenário pandêmico e dos protocolos sanitários que incidem na organização do atendimento na educação infantil do município.

A partir das discussões apresentadas ao longo deste artigo, foi possível constatar que a implementação de um sistema de avaliação da qualidade na educação infantil requer indissociabilidade entre política de avaliação e instrumento de avaliação.

A política de avaliação é abrangente - trata-se de um planejamento maior voltado para as políticas públicas, que garantem as ações por meio da legislação educacional - e inclui ainda a previsão de financiamento para a qualidade da oferta, a eficiência na gestão dos sistemas e, também, a formação docente. Para que uma política avaliativa seja eficiente, faz-se necessária uma rede de apoio por meio da intersetorialidade, que se refere às garantias previstas nas políticas públicas, no compromisso social com a educação e na necessidade de articulação e planejamento das diferentes instituições.

Quanto ao instrumento de avaliação, de abordagem contextual, sua finalidade é a de coletar, analisar e definir a qualidade da oferta, considerando a metodologia da avaliação. Além disso, tal instrumento propõe que o foco da avaliação seja baseado em insumos, ambientes e processo, visando a contemplar a especificidade institucional, bem como prevê abrangência em nível de sistema. Ademais, ele demarca a importância de considerar os ambientes físico e curricular com parâmetros de qualidade relativos à infraestrutura, aos materiais, à gestão dos serviços, às práticas pedagógicas e à garantia do direito da criança como a principal finalidade da unidade educativa; atrelados a isso estão os ambientes relacional e social com parâmetros que preveem a participação da comunidade educativa, ancorada na finalidade social que a instituição estabelece na comunidade em que oferta os serviços.

É pertinente salientar que Moro (2017) destaca a importância de um sistema de avaliação da qualidade da educação infantil que considere os ambientes físico, relacional e social, em que o instrumento enfatize as práticas pedagógicas e a relação com a comunidade educativa.

Ainda, o instrumento deve contemplar a gestão da avaliação, que envolve o estabelecimento de um plano de melhorias, com metas, prazos, responsáveis e a autoavaliação periódica para o monitoramento da gestão dos resultados.

Os elementos anteriormente descritos caracterizam a avaliação da educação infantil como um processo e não apenas como fim determinante do nível de qualidade. Cada elemento tem seu papel importante no mapeamento e na promoção da qualidade do atendimento.

\section{Considerações finais}

Este estudo identificou, descreveu e comparou os modelos de avaliação da qualidade na educação infantil de origem nacional e estrangeira. A análise conduzida mostrou como adequados os instrumentos avaliativos que se baseiam no acesso, insumos e processos, assim como os modelos que apresentam coerência metodológica são adaptáveis ao contexto avaliado e preveem a participação. Os instrumentos de avaliação que mais se aproximaram da realidade florianopolitana foram os Indicadores da qualidade na educação infantil e os instrumentos italianos de avaliação de contexto.

Quanto aos Indicadores da qualidade na educação infantil, os municípios de São Paulo, Salvador e o Distrito Federal já mostraram a adequação do instrumento, adaptando-o às suas propostas pedagógicas. Esse modelo de avaliação parte de um padrão nacional, representando um núcleo comum, mas também pode se adaptar aos elementos pertinentes ao contexto e 
às especificidades da unidade educativa. O referido instrumento apresenta potencialidade de promover a avaliação da qualidade da educação infantil de modo sistêmico e abrangente, considerando a necessidade de ter uma rede de apoio para a gestão dos resultados.

No que se refere à avaliação de contexto, pesquisas realizadas no Brasil, tais como as de Bondioli (2014), Souza et al. (2017) e Castro e Martins (2018), confirmam a pertinência e potencialidade dessa concepção avaliativa para promover a melhoria da qualidade da educação infantil brasileira. Tal estratégia avaliativa fomenta a participação e a reflexão em torno dos serviços ofertados para a infância (Souza et al., 2017). O desafio de uma avaliação de contexto é bastante amplo, pois abrange dimensões relacionais, pedagógicas e estruturais.

Um sistema de avaliação da qualidade na educação infantil deve possibilitar garantias, como, por exemplo, das políticas públicas de Estado, destinadas à for mação de professores e gestores educacionais, qualificando-os para o domínio dos processos e do instrumento de avaliação, assim como atribuindo responsabilidades aos gestores indiretos. Há de se considerar a possibilidade de cada município pensar e construir seu próprio instrumento de avaliação com base nas diretrizes que norteiam sua proposta pedagógica - tendo uma política de avaliação com suporte financeiro e de colaboração mútua entre a união e os entes federados (estados e municípios) -, com a assessoria técnica e pedagógica do MEC para uma avaliação sistêmica e integrada entre os municípios brasileiros para a adaptação do instrumento ao seu contexto.

\section{Referências}

Becchi, E., Bondioli, A., \& Ferrari, M. (2014). ISQUEN: Indicadores e escala de avaliação da qualidade educativa da creche. In L. Cipollone (Org.), Instrumentos e indicadores para avaliar a creche: Um percurso de análise da qualidade (pp. 149-197). UFPR.

Bondioli, A. (2009). ERVIS: Elementi per Rilevare e Valutare l'integrazione Scolastica. Editora Junior.

Bondioli, A. (2014). Indicadores operativos e análise da qualidade: Razões e modos de avaliar. In L. Cipollone (Org.), Instrumentos e indicadores para avaliar a creche: Um percurso de análise da qualidade (pp. 47-72). UFPR.

Bondioli, A., \& Sávio, D. (2013). O método. In A. Bondioli (Org.), Participação e qualidade em educação da infância: Percursos e compartilhamento reflexivo em contextos educativos (L. E. Fritoli, Trad., pp. 23-49). UFPR.

Campos, M. M., Esposito, Y. L., Bhering, E., Gimenes, N., \& Abuchaim, B. (2011). A qualidade da educação infantil: Um estudo em seis capitais brasileiras. Cadernos de Pesquisa, 41(142), 20-54.

Castro, J. S. de, \& Martins, A. F. (2018). Avaliação na e da educação infantil: Avaliação de contexto. Pro-Posiçôes, 29(2), 11-23.

Festa, M. (2019). Autoavaliação institucional participativa da educação infantil da cidade de São Paulo [Tese de doutorado, Universidade de São Paulo]. Biblioteca Digital de Teses e Dissertações da Universidade de São Paulo. https://teses.usp.br/teses/disponiveis/48/48134/tde-01082019-154513/pt-br.php

Gariboldi, A., \& Maselli, M. (2018). Construindo a avaliação em conjunto: A experimentação de uma abordagem participativa para avaliação na região italiana da Emília Romanha. Pro-Posições, 29(2), 46-71.

Harms, T. (2013). O uso de escalas de avaliação de ambientes na educação infantil. Cadernos de Pesquisa, 43(148), 76-97.

Harms, T., Cryer, D., \& Clifford, R. M. (2006). Infant/toddler environment rating scale. Teachers College Press. HighScope Educational Research Foundation. (2005). Child Observation Record (COR). Information for Decision Makers. High/Scope Press. 
Lein. 13.005, de 25 de junho de 2014. (2014). Aprova o Plano Nacional de Educação - PNE e dá outras providências. Brasília, DF.

Marcuccio, M., \& Zanelli, P. (2013). Sguardi sul nido... Strumento per lo Sviluppo di Processi Riflessivi e Indagini valutative nei Nidi da parte dei Gruppi di lavoro educativi (SPRING). Edizioni Junior-Spaggiari Edizioni.

Martínez Preciado, J. F. (2010). La construcción de indicadores y evaluación de la calidad en centros educativos: Seis experiencias en México. REICE: Revista Iberoamericana sobre Calidad, Eficacia y Cambio en Educación, 8(5), 133-153.

Ministério da Educação. (2006). Parâmetros nacionais de qualidade para a educação infantil (Vols. 1-2). $\mathrm{MEC} / \mathrm{SEB}$.

Ministério da Educação. (2009). Indicadores da qualidade na educação infantil. MEC/SEB. http://portal.mec.gov.br/dmdocuments/indic_qualit_educ_infantil.pdf

Ministério da Educação. (2010). Diretrizes Curriculares Nacionais para a Educação Infantil. MEC/SEB. http://portal.mec.gov.br/dmdocuments/diretrizescurriculares_2012.pdf

Ministério da Educação. (2011). Monitoramento do uso dos indicadores da qualidade na educação infantil. MEC/SEB. http://portal.mec.gov.br/index.php?option=com_ docman\&view=download\&alias=13119-relatorio-versao-internet-final-pdf-pdf\&category_ slug $=$ maio-2013-pdf\&Itemid $=30192$

Ministério da Educação. (2012). Educação infantil: Subsídios para a construção de uma sistemática de avaliação (Documento produzido pelo Grupo de Trabalho instituído pela Portaria n. 1.147/2011). MEC/SEB/Coedi. http://nepiec.com.br/producoes/Educacao\%20Infantil\%20sistematica\%20 de\%20avaliacao.pdf

Ministério da Educação. (2015). Contribuições para a política nacional: Avaliação em educação infantil a partir da avaliação de contexto. MEC/SEB/Coedi. http://primeirainfancia.org.br/wp-content/ uploads/2016/04/seb_avaliacao_educacao_infantil_a_partir_avaliacao_contexto.pdf

Moro, C. (2017). Avaliação de contexto e políticas públicas para a educação infantil. Laplage em Revista, 3(1), $44-56$.

Moro, C. (2018). Diferentes olhares para a creche: A avaliação de contexto com o instrumento SPRING em um município da Emilia Romagna. Revista Linhas, 19(40), 138-160.

Moro, C., \& Souza, G. de. (2016). Para uma análise pedagógica dos contextos educativos. (Entrevista com Anna Bondioli, Monica Ferrari, Donatella Savio). Universidade de Pávia/Itália.

Motiejunaite, A., Delhaxhe A., Balcon, M.-P., \& Borodankova, O. (2014). La educación y atención a la primera infancia. Agencia Ejecutiva en el Ámbito Educativo, Audiovisual y Cultural.

Myers, R. (2011). Em busca da qualidade educacional na pré-escola: Uma experiência mexicana. Cadernos de Pesquisa, 41(142), 100-115.

Pimenta, C. O. (2017). Avaliaçôes municipais da educação infantil: Contribuiçôes para a garantia do direito à educação das crianças brasileiras? [Tese de doutorado, Universidade de São Paulo - Faculdade de Educação].

Portaria n. 10, de 8 de janeiro de 2021. (2021). Estabelece parâmetros e fixa diretrizes gerais para implementação do Sistema de Avaliação da Educação Básica - Saeb, no âmbito da Política Nacional de Avaliação da Educação Básica. MEC/Inep.

Portaria n. 250, de 5 de julho de 2021. (2021). Estabelece as diretrizes de realização do Sistema de Avaliação da Educação Básica - Saeb no ano de 2021. MEC/Inep.

Portaria n. 271, de 22 de março de 2019. (2019). Estabelece as diretrizes de realização do Sistema de Avaliação da Educação Básica (Saeb) no ano de 2019. MEC/Inep.

Portaria n. 458, de 5 de maio de 2020. (2020). Institui normas complementares necessárias ao cumprimento da Política Nacional de Avaliação da Educação Básica. MEC. 
Portaria n. 1.147, de 16 de dezembro de 2011. (2011). Institui Grupo de Trabalho de Avaliação da Educação Infantil. MEC.

Secretaria de Estado de Educação. (2019). Indicadores de qualidade da educação infantil. SEE.

Secretaria Municipal da Educação. (2016). Indicadores da qualidade na educação infantil da rede municipal de ensino de Salvador. (Versão para a Primeira Aplicação (abril/2016)). Nossa Rede - Projeto Pedagógico de Salvador. Salvador. http://educacao.salvador.ba.gov.br/pdfs-nossa-rede/documentosmunicipais/educacao-infantil/profissionais/indique\%20Salvador\%20vers\%C3\%A3o\%20 FINAL\%202016\%20rev.pdf

Secretaria Municipal de Educação. (2016). Indicadores de qualidade da educação infantil paulistana. DOT. São Paulo. https://www.sinesp.org.br/images/9___INDICADORES_DE_QUALIDADE_NA_ EDUCACAO_INFANTIL_PAULISTANA.pdf

Sousa, S. Z., \& Pimenta, C. O. (2018). Avaliação como integrante de políticas públicas de educação infantil. Pátio Educação Infantil, 16(57), 4-7.

Souza, G. de, Moro, C., \& Coutinho, A. S. (2015). Formação da rede em educação infantil: Avaliação de contexto. Appris.

Souza, G. de, Moro, C., França, F. F., \& Rodrigues, A. J. L. (2017). A pesquisa em rede na educação infantil: Avaliação de contexto, modos de proceder e possibilidades de reflexão. RELAdEI: Revista Latinoamericana de Educación Infantil - Evaluación de Contextos en Educación Infantil, 6(1-2), 23-32.

Tayler, C. (2014). Avaliação da qualidade da educação infantil na Austrália. Estudos em Avaliação Educacional, 25(58), 126-151.

United Nations Educational, Scientific and Cultural Organization. (2017). MELQO - Measuring Early Learning Quality and Outcomes. United Nations Educational, Scientific and Cultural Organization Overview, (p. 112). https://www.brookings.edu/wp-content/uploads/2017/06/melqo-measuringearly-learning-quality-outcomes.pdf

\section{Nota sobre autoria}

Dirce Guerres-Zucco - escrita do manuscrito original, revisão e edição; Andreia Zanella - revisão e edição da escrita, supervisão; Angela Scalabrin Coutinho - revisão e edição da escrita.

\section{Disponibilidade de dados}

Os dados subjacentes ao texto da pesquisa estão informados no artigo.

\section{Como citar este artigo}

Guerres-Zucco, D., Zanella, A., \& Coutinho, A. S. (2022). Instrumentos de avaliação e parâmetros de qualidade para a educação infantil. Cadernos de Pesquisa, 52, Artigo e07958. https://doi.org/10.1590/198053147958 